\title{
ARTICLE
}

Received 8 Apr 2013 | Accepted 5 Aug 2013 | Published 13 Sep 2013 DOl: 10.1038/ncomms3401

\section{Evidence of two distinct local structures of water from ambient to supercooled conditions}

\author{
A. Taschin ${ }^{1}$, P. Bartolini ${ }^{1}$, R. Eramo ${ }^{1,2}$, R. Righini ${ }^{1,3} \&$ R. Torre ${ }^{1,4}$
}

The liquid and supercooled states of water show a series of anomalies whose nature is debated. A key role is attributed to the formation of structural aggregates induced by critical phenomena occurring deep in the supercooled region; the nature of the water anomalies and of the hidden critical processes remains elusive. Here we report a time-resolved optical Kerr effect investigation of the vibrational dynamics and relaxation processes in supercooled bulk water. The experiment measures the water intermolecular vibrations and the structural relaxation process in an extended temperature range, and with unprecedented data quality. A mode-coupling analysis of the experimental data enables to characterize the intermolecular vibrational modes and their interplay with the structural relaxation process. The results bring evidence of the coexistence of two local configurations, which are interpreted as high-density and low-density water forms, with an increasing weight of the latter at low temperatures.

\footnotetext{
${ }^{1}$ European Lab for Non-Linear Spectroscopy (LENS), Univ. di Firenze, via N. Carrara 1, Sesto Fiorentino, I-50019 Firenze, Italy. ${ }^{2}$ Istituto Nazionale Ottica, CNR, Largo Fermi 6, I-50125 Firenze, Italy. ${ }^{3}$ Dip. di Chimica, Univ. di Firenze, via Della Lastruccia 13, Sesto Fiorentino, I-50019 Firenze, Italy. ${ }^{4}$ Dip. di Fisica e Astronomia, Univ. di Firenze, via G. Sansone 1, Sesto Fiorentino, I-50019 Firenze, Italy. Correspondence and requests for materials should be addressed to R.T. (email: torre@lens.unifi.it).
} 
W ater can remain liquid below its melting point and stays in its metastable phase known as supercooled water ${ }^{1,2}$. Several thermodynamic (for example, isothermal compressibility, isobaric heat capacity and thermal expansion coefficient) and dynamic (for example, viscosity, diffusivity, structural relaxation) observables of liquid water show an anomalous behaviour ${ }^{3}$. In the supercooled state, the values of these physical observables clearly diverge, with a singularity temperature $T_{\mathrm{s}}$ estimated at $\simeq 228 \mathrm{~K}$. Despite the wide interest and the numerous research efforts on this subject, the origin of these critical phenomena remained unclear ${ }^{4-6}$ and are lively debated ${ }^{7-14}$.

It is widely accepted that the water anomalies are connected with the formation of various structural aggregates whose features are modified by the temperature/pressure conditions ${ }^{3}$. A crucial point is the physical origin of these structural aggregations.

In their pioneering work, Poole et al. ${ }^{15}$ postulated the existence of a liquid-liquid critical point (LLCP) based on a simulation study and on the experimental observation of polyamorphism in glassy water (that is, the existence of two forms of water glass: low-density and high-density amorphous ice). In the LLCP hypothesis, a first-order phase transition takes place involving two supercooled water liquid forms: the low-density (LD) and the high-density (HD) phases. The LD phase is characterized by tetrahedral intermolecular coordination, whereas the $\mathrm{HD}$ one shows more packed structures with distorted networks. The existence of an LLCP is compatible with a series of computational and experimental results ${ }^{3}$. Recent new simulation studies confirmed the LLCP hyphotesis ${ }^{10-14}$. Nevertheless, the matter is still under debate: in their recent simulation study, Limmer and Chandler ${ }^{9}$ interpreted the water anomalies as non-equilibrium phenomena associated to the liquid-crystal transition. Moreover, the relevance of ice nucleation phenomena and their relationship with the water anomalies has been reported ${ }^{7,8}$.

A fundamental question to explain the origin of water anomalies is if two distinct intermolecular local and transient configurations, whose densities are different, persist at temperature higher than $T_{s}$. The fluctuations between these two water species could explain the thermodynamic and dynamic anomalies. Unfortunately, the most evident critical phenomena are expected to occur in the supercooled phase at temperature/ pressure values not directly accessible in bulk water samples, and this prevents a direct experimental solution of the problem. In fact, in several experimental and simulation works, the water singularity temperature $T_{\mathrm{s}}$ at atmospheric pressure has been extrapolated to be around $223-228 \mathrm{~K}$, whereas homogeneous nucleation fixes the lowest reachable temperature at about $231 \mathrm{~K}$ for liquid water. In fact, in a macroscopic bulk sample, the crystallization phenomena and several experimental difficulties limit the minimum temperature around $243 \mathrm{~K}$. Nano-confinement has the advantage of preventing water freezing and of extending the experimental investigation of the supercooled phase down to very low temperatures; it is, however, still unclear to what extent the interactions with the pore surfaces modify the water properties ${ }^{16-20}$. The hydrogen bond network largely determines the intra- and intermolecular vibrational spectrum of supercooled water; thus, the experimental investigation of the spectral features gives precious information on the local intermolecular structures and on their variation with the temperature/pressure conditions. The investigation of highfrequency spectrum, $1,000-4,000 \mathrm{~cm}^{-1}$, gives access to the intramolecular vibrational dynamics that has been recently related to the LLCP hypothesis ${ }^{21}$. The low-frequency spectrum of water, $v<1,000 \mathrm{~cm}^{-1}$, reflects the intermolecular dynamics, and hence it is particularly sensitive to the local molecular structures. This frequency range of the water spectrum has been investigated in several light-scattering studies (see for example, refs 22-27 and references at http://www.lsbu.ac.uk/water/). Two main broad peaks are observed at 50 and $200 \mathrm{~cm}^{-1}$, generally attributed to the hydrogen bond 'bending' and 'stretching' vibrations. The spectra show also a very low-frequency wing, $v<20 \mathrm{~cm}^{-1}$, which has been attributed to 'relaxation' processes. The light-scattering data were measured at relatively high temperatures and have rather poor signal-to-noise ratio because of the very weak water signal: these drawbacks did not permit to identify all the possible vibrational modes possibly contributing to the water spectrum in this region. Moreover, the reported attempts of fitting the lightscattering data have been based on a simple superposition of independent Gaussian and Lorentzian functions, neglecting any possible coupling between the vibrational dynamics and the structural relaxation processes.

An accurate analysis of the low-frequency spectral features of supercooled water, including a quantitative comparison with the predictions of recent models for water dynamics is still missing; it actually appears as a required step towards the full understanding of the structural and dynamical properties of water in its lowtemperature metastable state.

In recent years, time-resolved non-linear spectroscopy has been demonstrated to be a very useful tool for the investigation of complex liquid dynamics ${ }^{28}$. Several studies of this type have been performed on liquid water; only in few cases have they been extended to its supercooled phase ${ }^{29-32}$. Time-resolved optical Kerr effect (OKE) investigations of liquid water ${ }^{29,33-36}$ show the signature of fast vibrational dynamics at short times, followed by a slower monotonic relaxation. The initial oscillatory component provides information about intermolecular hydrogen bond dynamics, and can be directly compared with the low-frequency Raman spectrum ${ }^{33}$. In fact, the OKE experiments on supercooled water ${ }^{29}$ have been focused so far on the investigation of the slower decay, which is interpreted as a structural relaxation phenomenon. No detailed OKE investigation of the vibrational dynamics in supercooled water has been carried out because of the intrinsic experimental difficulties; hence, a detailed picture of this dynamics is still missing ${ }^{37}$.

Here, using an improved experimental set-up ${ }^{31}$, we measure, for the first time according to our knowledge, the fast vibrational dynamics and the slow structural relaxation in a single data set characterized by low noise and wide dynamic range. Moreover, we reached temperatures lower than in previous experiments, approaching the homogeneous nucleation limit. We succeed in measuring the OKE signal relaxation over a large time interval of about 25 ps with a high time resolution, better than $20 \mathrm{fs}$. We thus provide the unambiguous measurement of the entire correlation function in supercooled water, enabling a very stringent comparison with the available theoretical models.

\section{Results}

Experimental techniques. Time-resolved OKE spectroscopy is a non-linear technique exploiting the transient birefringence induced by the electric field of radiation in an optically transparent and isotropic medium. The method has been described in many papers and review articles ${ }^{38-40}$. In short, the oscillating electric field of a polarized short laser pulse (the pump) induces optical birefringence in the sample. In addition to the instantaneous electronic contribution (which does not carry any dynamic information), the induced birefringence reflects the relaxation and vibrational response of the molecules in the sample. A second pulse (the probe), spatially superimposed within the sample with the pump pulse and characterized by a different polarization, probes the induced birefringence. The intensity of probe light leaking through a polarizer placed after 
the sample measures the material response as a function of the time delay between the pump and the probe.

If femtosecond laser pulses are employed, the method enables to measure the relaxation processes and the low-frequency vibrational correlation functions of the system. In this sense, it is the time-domain counterpart of depolarized light-scattering technique ${ }^{39,40}$. The wide accessible time window, extending from tens of femtoseconds to hundreds of nanoseconds, makes OKE a very flexible technique, capable of revealing very different dynamic regimes, from simple liquids to supercooled liquids and glass formers ${ }^{41-46}$. In the case of optical heterodyne detection, the delay time-dependent signal is directly proportional to the material response (relaxation) function, $R(t)$, convoluted with the instrumental function, $G(t)$ (see Methods). The response $R(t)$ is directly connected to the time derivative of the time-dependent correlation function of the anisotropic component of the collective susceptibility ${ }^{39,40,44,47}$ :

$$
R(t) \propto-\frac{\partial}{\partial t}\langle\chi(t) \chi(0)\rangle
$$

the imaginary part of the Fourier transform of $R(t)$ corresponds to the frequency-dependent response, the observable measured in depolarized light-scattering (DLS) experiments ${ }^{48}$. We stress here that measuring the correct instrumental function $G(t)$ is a crucial step for achieving a reliable and accurate fitting of the experimental OKE signal. A detailed description of the method adopted is given in the Methods section.

Supercooling bulk water can be achieved only with particularly pure sample and using specific attention for the cooling procedure. We performed the measurements on a sealed vial of cylindrical shape, prepared for pharmaceutical purposes by the Angelini company, which allowed us to reach the temperature of $247 \mathrm{~K}$. The sample temperature was controlled by means of a home-made cryostat with a stability of $0.1 \mathrm{~K}$, using a Peltier cooler.

Measured data. The heterodyne-detected OKE (HD-OKE) data are reported in Fig. 1: the data clearly show the complex dynamic features presented by liquid and supercooled water. The vibrational dynamics, extending up to $1 \mathrm{ps}$, displays an articulate pattern that becomes progressively more defined, going deep in the supercooled phase, and which at longer times merges into the monotonic decay due to structural relaxation.

The time-resolved HD-OKE experiment measures the timedependent correlation function of the water susceptibility (that is, collective polarizability), see equation (1). The time evolution of this function reflects the water dynamics through the electronic polarizability. Numerical investigations ${ }^{37,47,49}$ show that the OKE signal of water is the result of a complex interplay between intrinsic molecular terms and interaction-induced contributions. These calculations reveal how the fluctuations of the water susceptibility, including the anisotropic components, are largely determined by the intermolecular translational dynamics. This behaviour is expected, given the essentially isotropic water molecular polarizability. The density fluctuations (that is, translational dynamics) dominate the relatively low-frequency range of the density-of-states ${ }^{50,51}, v<400 \mathrm{~cm}^{-1}$, whereas the rotational/librational dynamics affect the highfrequency spectrum ${ }^{52}, v>400 \mathrm{~cm}^{-1}$. Moreover, the structure and dynamics of the $\mathrm{H}$-bond network are fundamental properties that affect the susceptibility fluctuations at any frequency.

The relaxation features presented by supercooled water are very similar to those of glass-forming liquids ${ }^{43}$, and the slow dynamics is found in agreement with the main mode-coupling (MC) theory predictions ${ }^{29}$. In Fig. 2 we report the master plot analysis of the slow component of the HD-OKE signal decay.

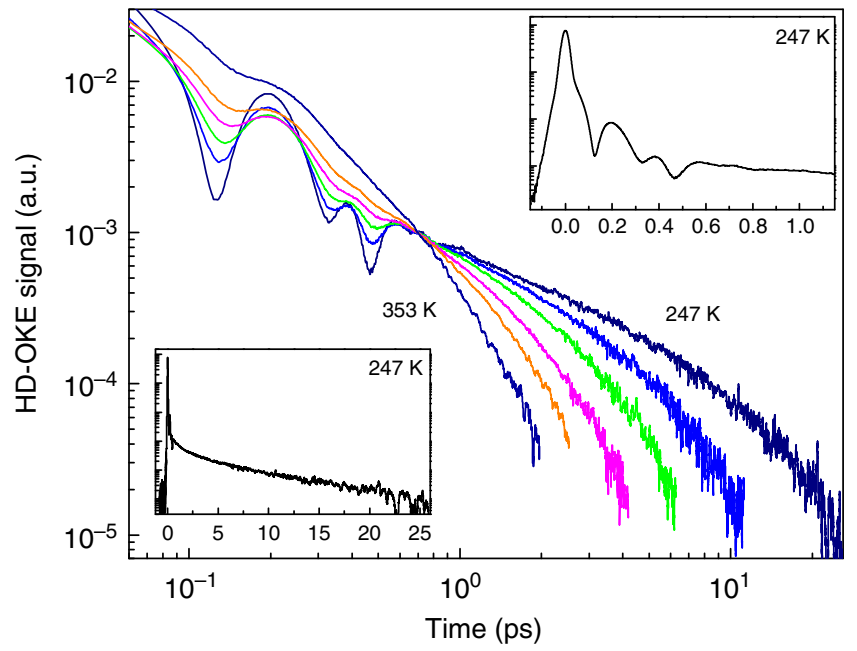

Figure 1 | HD-OKE signal from liquid and supercooled water. In the main panel, the experimental signal is reported as a function of temperature in a log-log plot. At short delay times ( $<1 \mathrm{ps}$ ), the HD-OKE signal shows oscillations due to vibrational dynamics, which become more defined as the temperature is lowered below the melting point. At longer delay times, the signal is characterized by a monotonous decay, which is strongly temperature dependent and clearly non-exponential. For a readable display, only six temperatures $(247,258,273,293,313$ and $353 \mathrm{~K})$ out of the 11 measured (the previous ones plus 253, 263, 268, 278 and $333 \mathrm{~K}$ ) are reported; the intensities are normalized at $0.7 \mathrm{ps}$. The upper and lower insets show, in a log-linear plot, the $247 \mathrm{~K} \mathrm{HD}$-OKE data at short- and long delay times, respectively.

The new data confirm, over an extended temperature range, the power-law temperature dependence of the relaxation time. Other experimental investigations reported similar power-law temperature dependence of different water observables ${ }^{3}$, both static and dynamic; recently, a small-angle X-ray scattering investigation ${ }^{53}$ also found that the correlation length of the density fluctuations follows a power law with similar value of $T_{\mathrm{c}}$.

Data analysis and fits. The analysis of the whole time-dependent correlation function measured by the optical experiments requires the utilization of a model that enables an operative parametrization of the vibrational modes present in the water dynamics. This turns out to be a complex task, and different attempts have been pursued ${ }^{34-36}$ in that direction. None of these water studies was extended into the supercooled state. Moreover, the data analysis and the fitting models adopted so far did not include any coupling between the fast vibrational dynamics and the slow structural relaxation. We tested some of these fitting procedures on our HD-OKE data and found that they fail to reproduce correctly the HD-OKE data in the supercooled temperature range. To analyse the vibrational dynamics and the structural relaxation, including their coupling processes, we utilized the schematic solution of MC theory ${ }^{54}$.

The formalism of MC theory stems from a generalization of the Mori and Zwanzig hydrodynamic theory ${ }^{54}$. In the MC theory, the retardation effects are taken into account by memory functions $K(t)$, which have the function of the hydrodynamic friction coefficients. Of course, the task of any microscopic theory is that of providing the form of $K(t)$. In its simplest form, developed for simple atomic liquids, only density fluctuations contribute to memory; to overcome the limitations of this approach and to extend the MC theory description to the dynamics of more complex systems, several versions of the theory have been 

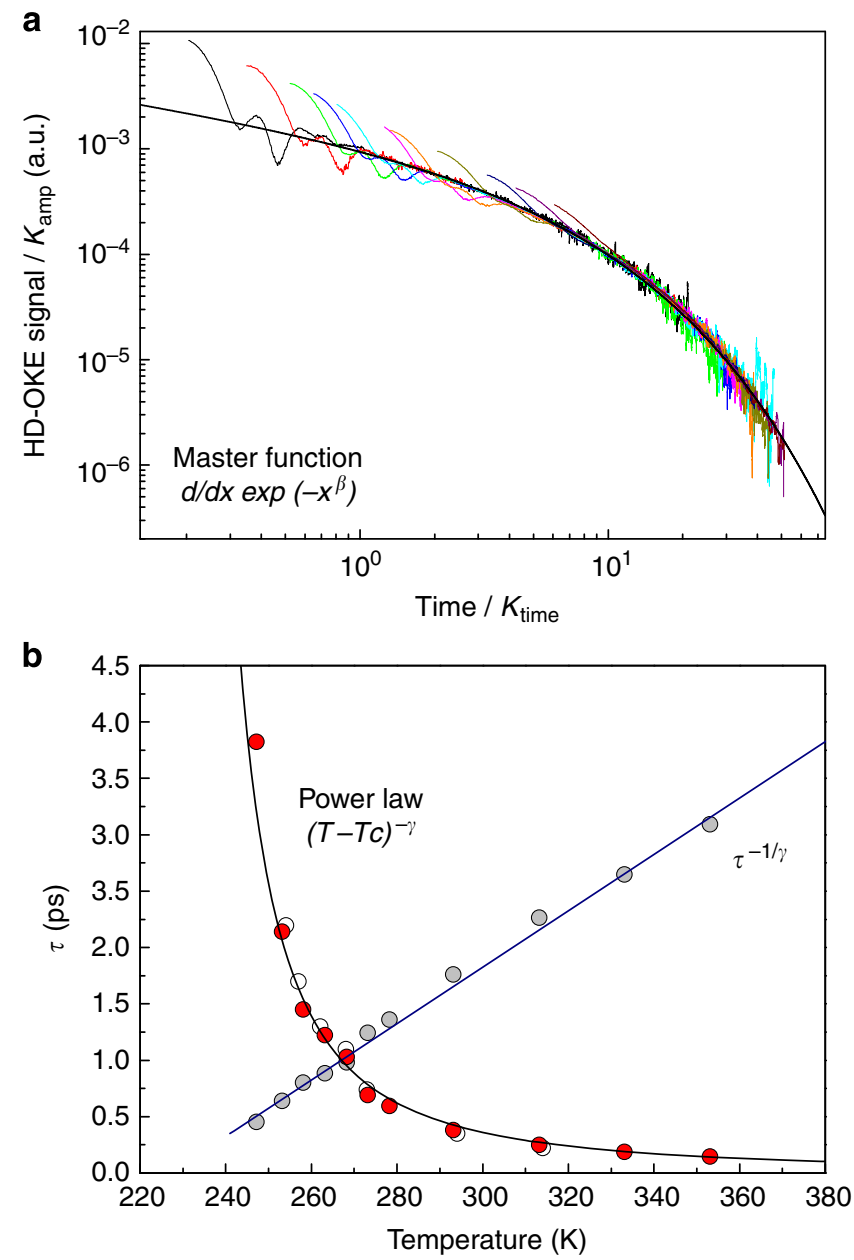

Figure 2 | Master plot and scaling law. The slow dynamics of supercooled water can be analysed using a model-independent procedure ${ }^{29}$, consisting in rescaling the time/amplitude scales of the raw data measured at different temperatures in a way that the decay curves overlap. If all the data collapse in a 'master plot', it is possible to identify a function, the 'master function', which defines the relaxation independently of the temperature ${ }^{54}$. In the panel a, we report the master plot of the HD-OKE data and the master function: $\frac{d}{d x} \exp \left(-x^{\beta}\right)$ with stretching parameter $\beta=0.6$. The relaxation times obtained with the rescaling procedure are shown in $\mathbf{b}$. The new data (red circles) extend and confirm the previous data (empty circles $)^{29}$. The temperature dependence of these data are in agreement with the power law: $\tau \propto\left(T-T_{\mathrm{c}}\right)-\gamma$, with critical temperature $T_{\mathrm{c}} \simeq 227 \mathrm{~K}$ and critical exponent $\gamma \simeq 1.7$. We report the relaxation times and the power law in a linearized plot to visualize the scaling proprieties and the critical temperature.

developed. For molecular liquids, in fact, orientational and intramolecular degrees of freedom contribute to the overall dynamics. In our analysis, we adopt the multi-component schematic formulation of the MC theory (SMC) ${ }^{55-57}$. Briefly, in this approach the density-density time correlator, $\Phi_{\mathrm{m}} \propto$ $\langle\rho(t) \rho(0)\rangle$, termed the master correlator, is described by the differential equation ${ }^{55}$ :

$$
\ddot{\Phi}_{\mathrm{m}}(t)+\eta_{\mathrm{m}} \dot{\Phi}_{\mathrm{m}}(t)+\Omega_{\mathrm{m}}^{2} \Phi_{\mathrm{m}}(t)+\int_{0}^{t} K\left(t-t^{\prime}\right) \dot{\Phi}_{\mathrm{m}}\left(t^{\prime}\right) d t^{\prime}=0,
$$

where $\Omega_{\mathrm{m}}$ is the mode frequency, $\eta_{\mathrm{m}}$ is the friction coefficient and the memory function $K(t)=v_{1} \Phi_{\mathrm{m}}(t)+v_{2} \Phi_{\mathrm{m}}^{2}(t)$ is expressed as a series expansion of the master correlator itself. The role of other observables, $A_{\mathrm{i}}$, linked to the density correlator dynamics (for example, the molecular orientation, the dynamics of a target molecule and so on) is described by a set of differential equations of the same form as equation (2), written in terms of slave correlators $\Phi_{\mathrm{i}}(t) \propto\left\langle A_{\mathrm{i}}(t) A_{\mathrm{i}}(0)\right\rangle$ and of memory functions of the form $m_{\mathrm{i}}(t)=v_{\mathrm{i}}^{\mathrm{s}} \Phi_{\mathrm{m}}(t) \Phi_{\mathrm{i}}(t)$, which implies the coupling of the master and slave correlators. The key point in the SMC analysis of the OKE experimental data is that the time correlation function of the sample polarizability can be expressed as the sum of the time derivatives of the slave correlators:

$$
R^{\mathrm{SMC}}(t) \propto-\frac{\partial}{\partial t} \sum_{\mathrm{i}} a_{\mathrm{i}} \Phi_{\mathrm{i}}(t) .
$$

We underline here that the individual slave correlators describe 'average collective modes', so that they cannot be associated to specific physical variables. In summary, the SMC approach represents a robust physical model capable of describing a complex dynamics, with no need of unjustified assumptions concerning the decoupling of slow and fast dynamics.

We solved the SMC equations numerically, taking the frequencies, friction and coupling coefficients as parameters to be adjusted to reproduce the HD-OKE response by a fitting procedure. The details on the SMC equations and fitting procedures are reported in the Methods section.

In Fig. 3, we show some of the measured HD-OKE signals with the fits obtained using the SMC model. The model reproduces correctly the experimental data over the whole time range at all temperatures. Most remarkably, and differently from other fitting models widely used, this result is achieved without imposing any non-physical decoupling of vibrational and relaxation dynamics.

An important result of our work is that the fitting at temperatures $\leq 313 \mathrm{~K}$ requires three slave correlators, whereas for higher temperatures only two correlators are needed. We would like to stress out that the master correlator alone cannot reproduce the data, suggesting that the density fluctuations are not enough to describe completely the dynamics of collective water polarizability; as expected, the hydrogen-bound dynamics adds no trivial dynamical components that determine the water susceptibility fluctuations.

The time-domain data, collected in the time-resolved HD-OKE experiments, are transformed to yield the corresponding spectra in the frequency domain. In fact, the Fourier transform of the HDOKE response function, $\tilde{R}(\omega)$, is directly connected with the spectrum $S_{\text {DLS }}(\omega)$ measured in the DLS experiments: the imaginary part of $\tilde{R}(\omega)$ is proportional to $S_{\mathrm{DLS}}(\omega)$ corrected for the Bose factor, that is, $\operatorname{Im}[\tilde{R}(\omega)] \propto S_{\mathrm{DLS}}(\omega) /[n(\omega)+1]$ (refs 39,40,48). Our results can then be directly compared with the DLS, Rayleigh and Raman data, found in the literature. The imaginary part of the frequency domain response function, $\operatorname{Im}[\tilde{R}(\omega)]$, was obtained as the Fourier transform of the HD-OKE signal deconvoluted from the instrumental function: $\operatorname{Im}[\tilde{R}(\omega)] \propto \operatorname{Im}\{F T[S(t)] / F T[G(t)]\}$. The Fourier transform of the measured HD-OKE response and of the calculated SMC response are reported in Fig. 4.

\section{Discussion}

The experimental spectra clearly show the characteristic vibrational features of liquid water: the two broad bands around 50 and $200 \mathrm{~cm}^{-1}$, and the low-frequency wing. This wing corresponds to the slow decay of the OKE signal measured in the time domain; as discussed in our previous work ${ }^{29}$, it is attributed to structural relaxation.

The other two bands are generally attributed to translational intermolecular modes (that is, the oscillatory dynamics of water molecules around their instantaneous centre of mass) hindered by the first-neighbour molecular cage ${ }^{49-51}$. In the liquid phase, they are largely affected by the hydrogen bonds. A comparison with 
a

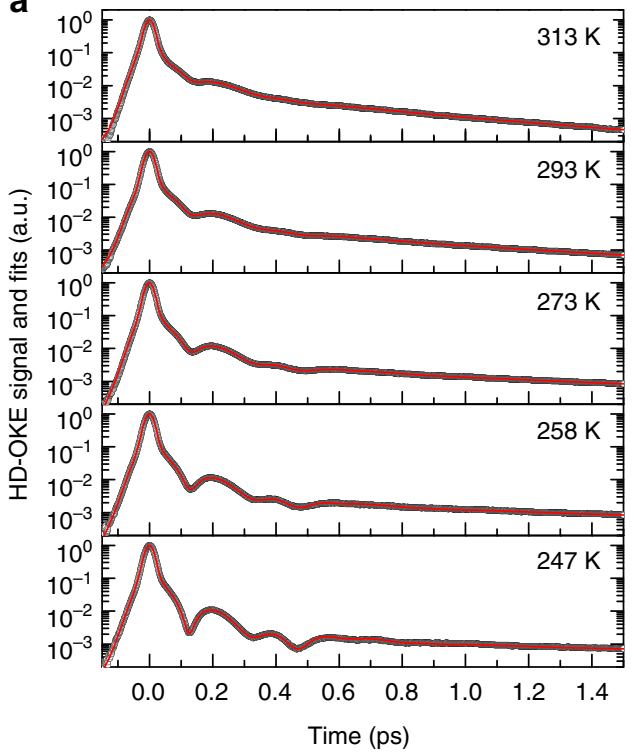

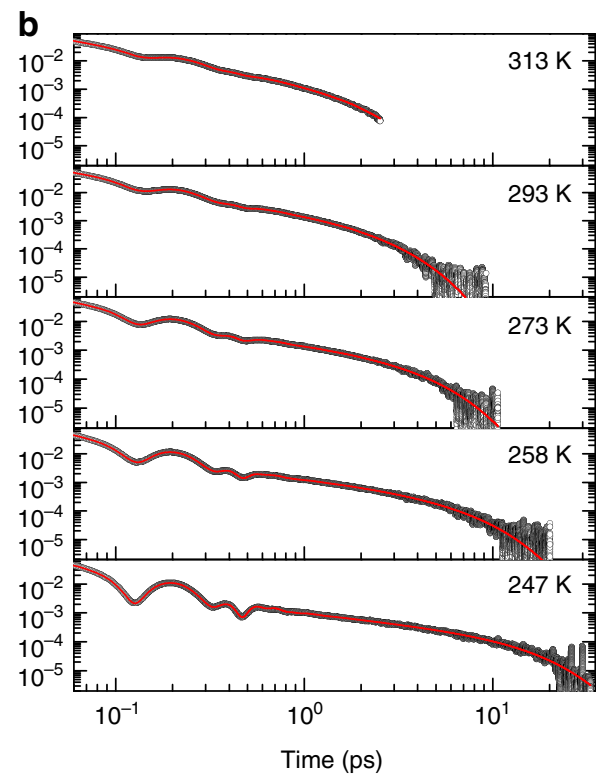

Figure 3 | Data analysis and fit based on the SMC theory. The complex vibrational dynamics taking place in the sub-picosecond time scale is correctly reproduced by the numerical solution of the SMC model. The SMC model enables a very good fit of the slow relaxation decays, corresponding to the stretched exponential function. In addition, at intermediate delay times, where the vibrational dynamics merge into the relaxation processes, the decay curve is correctly described by the SMC equations. We report the SMC fits (red line) of the HD-OKE data (circle) for five temperatures. In (a), the short time window is shown in a log-linear plot; in (b), the whole time window is shown in a log-log plot.

the calculated vibrational density-of-states reported in ref. 51 suggests specific assignment of the 50 - and the $200-\mathrm{cm}^{-1}$ bands. The first one is dominated by the transverse translational motion, whereas the second has essentially longitudinal character ${ }^{51}$. The physical interpretation is that the two intermolecular vibrations are mainly involving 'bending' and 'stretching' distortion of the hydrogen bonds, respectively ${ }^{24}$. This picture, even if intuitively useful, is clearly oversimplified: it does not take into account the coupling of the two vibrations imposed by the complex hydrogen bond network present in the liquid water. The presence of two different local structures, the one corresponding to highly tetrahedral intermolecular structures and that consisting of strongly distorted network, as well as their continuous reorganization induced by the structural relaxation processes, are expected to largely affect the vibrational dynamics.

The SMC fit enables to disentangle the vibrational modes contributing to this dynamics and to point out their coupling to the structural relaxation processes. The stretching mode, around $200 \mathrm{~cm}^{-1}$, needs two correlators to be properly reproduced. One correlator, peaked at about $180 \mathrm{~cm}^{-1}$ (blue line), shows an almost temperature-independent peak amplitude and a large spectral component extending to very low frequency, indicative of strong coupling to the structural dynamics. The other correlator, peaked at about $225 \mathrm{~cm}^{-1}$ (orange line), appears to be substantially uncoupled to the structural dynamics; its amplitude increases with decreasing temperature. Our HD-OKE measurement represents the first clear experimental determination of this feature, even if it was inferred in a previous Raman study ${ }^{22}$. The bending mode at about $50 \mathrm{~cm}^{-1}$ can be described by a single correlator (magenta line in Fig. 4) that turns out to be decoupled from the structural relaxation (the peak close to 0 frequency). It is possible that also the bending peak develops at low temperature a second component, but very likely the frequency separation is not sufficient to be resolved from our data analysis.

A comparison between these results and the recent debate on the nature of water anomalies is due. Our data and analysis show the presence of two distinct modes responsible for the part of the water spectrum normally attributed to the intermolecular stretching vibrations. Raman spectra of the LD and HD amorphous ice (LDA and HDA, respectively) in this frequency region differ from that of liquid water ${ }^{58}$. LDA has a broad peak centred at higher frequency, about $220 \mathrm{~cm}^{-1}$; HDA shows an even broader peak centred at about $185 \mathrm{~cm}^{-1}$ (see, for example, Fig. 1 in ref. 58), whereas the crystalline ice phases typically have a narrow peak at $230 \mathrm{~cm}^{-1}$. This suggests that the modes observed in our experimental investigations can be associated with two fluctuating water species with different local structures; a LD form characterized by a tetrahedral network and fourcoordinated molecules similar to the LDA structure, and an HD form characterized by closely packed aggregates with lower coordination and high network distortions. The mode at $225 \mathrm{~cm}^{-1}$ can be attributed to the LD form and the mode at $180 \mathrm{~cm}^{-1}$ to the HD one. The present data strongly support the existence of the LD and HD water species. Nevertheless, as the observed water dynamic is measured at temperature/pressure relatively far from the supposed LLCP, some ambiguity remains about the nature of the critical phenomena taking place into the supercooled phase. The spectroscopic features attributed to the LD and HD forms could be attributed either to the coexistence of two extended liquid phases leading to the liquid-liquid phase transition ${ }^{1,3,15}$, or to the continuous transition from a normal, unstructured liquid to a tetrahedrally structured liquid ${ }^{7}$.

Our new experimental data and analysis clearly show the presence of the high-frequency satellite mode contributing to the intermolecular stretching band of water, whose amplitude increases noticeably when the sample is cooled into the supercooled metastable phase. The nature of this mode, peaked at $225 \mathrm{~cm}^{-1}$, appears clearly different from that of the nearby mode at $180 \mathrm{~cm}^{-1}$. In fact, the former is strongly temperature dependent and only weakly coupled to the structural relaxation processes, whereas the latter is almost temperature independent and strongly coupled to structural rearrangements. The surprising similarities of the spectra of LDA and HDA glass forms with those of the two high-frequency distinct modes, reported in this 


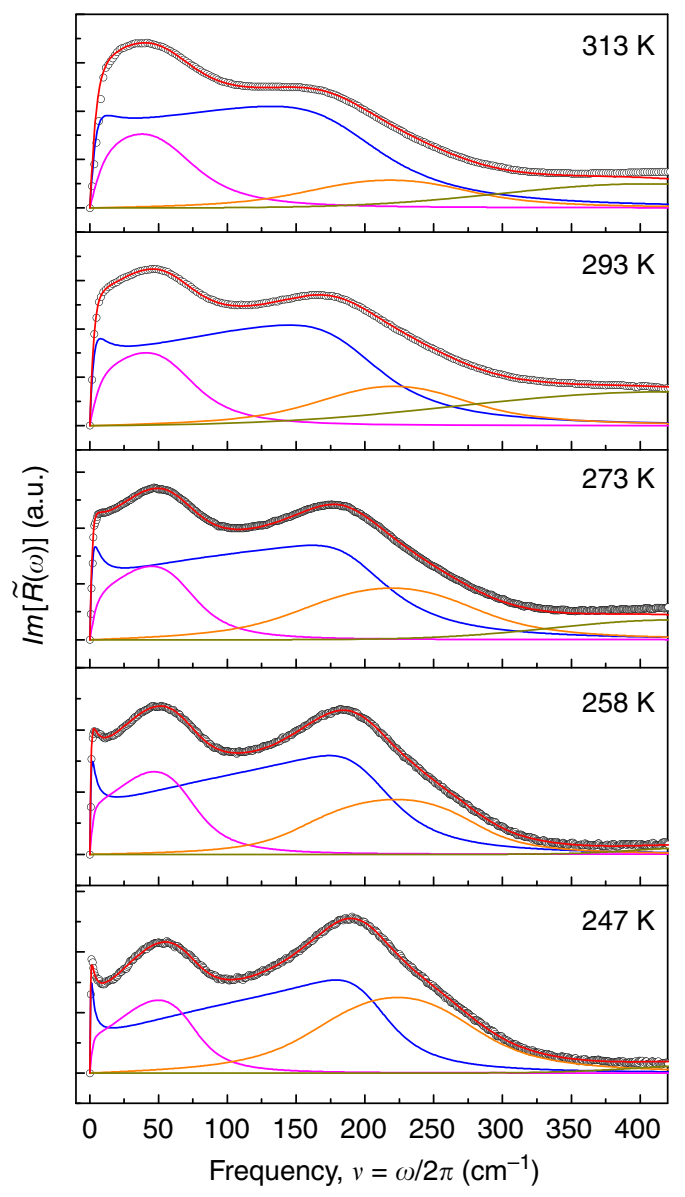

Figure 4 | Water spectrum and vibrational components. The Fourier transform of the time-dependent OKE response, $\tilde{R}(\omega)$, is directly related to the frequency-dependent spectrum measured in the dynamic lightscattering experiments. The imaginary part of the Fourier transform of the HD-OKE data (open circle), after which the contribution of the instrumental function has been removed by de-convolution, and of SMC fits (red line) at $247,258,273,293$ and $313 \mathrm{~K}$ are reported. The SMC model enables to disentangle the dynamical features that generate the simulated response function. The contributions of the three slave correlators are reported in the figure as magenta-blue-orange lines. Moreover, a simple damped harmonic oscillator (dark yellow) has been added to the SMC response to reproduce the very high-frequency vibrational contributions $\left(v>400 \mathrm{~cm}^{-1}\right)$; this does not affect the response function in the lower frequency range, where the dynamics relevant to our investigation takes place. The simulation of HD-OKE data by SMC model requires two vibrational modes to fit the intermolecular stretching band of water (blue and orange lines). The characteristics of these two modes are clearly different in terms of spectrum shape and temperature dependence.

work, suggest attributing them to the LD and HD water structures, respectively. The LD water forms are expected to consist of aggregates of a small number of molecules; being mostly localized in space (at least in the temperature range investigated), their dynamics show negligible coupling with the structural relaxation. The two local configurations of liquid water coexist in the entire temperature interval considered, above and below the melting temperature. The number/lifetime of the LD species must increase with decreasing temperature, to account for the amplitude increase of the corresponding correlator revealed by our analysis. On the other hand, the vibrational dynamics of the fraction of HD water appears dominated by the bare caging effect that merges into the structural relaxation, a behaviour typical of fragile glass-former liquids.

We end by remarking that our findings are consistent with the conclusion drawn recently by Nilsson et al. ${ }^{59}$ on the basis of $\mathrm{X}$-ray emission and absorption experiments, and by Overduin and Patey ${ }^{60}$ from the analysis of their computer simulations. In both papers, large-density fluctuations in liquid water are attributed to a short-range bimodal aggregation of molecules with disordered and tetrahedral local environments.

\section{Methods}

Water sample. Supercooling bulk water is not an easy task because of crystallization. Temperatures as low as 20 degrees below the melting point can be achieved only with particularly pure sample and using specific attention for the cooling procedure. In our work, we performed the measurements on bi-distilled water prepared by the Angelini company in a sealed vial of cylindrical shape, which enables us to reach a temperature as low as $247 \mathrm{~K}$. The sample temperature is controlled with a stability of $0.1 \mathrm{~K}$ by means of a home-made cryostat. The vial is inserted into an aluminum holder, with a central cylindrical housing whose diameter is close to that of the vial. Two fused silica windows on opposite sides of the aluminum holder allow the beams to cross the sample. A thin film of glycerol between the vial and the housing assures an efficient heat transfer. The holder is fixed to the cold plate of a Peltier cooler and the temperature is controlled by a platinum thermoresistance in thermal contact with the holder itself.

HD-OKE set-up. We used the optical configuration introduced by Giraud et al. ${ }^{61}$, which enables a reliable and efficient optical heterodyne detection of the OKE signal; a scheme of the optical set-up is shown in Supplementary Fig. S1. The laser system utilized is a self-mode-locked Ti:Sapphire laser (by Femtolasers, mod. Fusion) producing pulses of $20 \mathrm{fs}$ duration with an energy of $3 \mathrm{~nJ}, 77.47 \mathrm{MHz}$ repetition rate and $800 \mathrm{~nm}$ wavelength. Other details on the experimental set-up are reported in refs 31,62 .

HD-OKE signal. The signal measured in an HD-OKE experiment is ${ }^{63-65}$ :

$$
S(t) \propto \int_{-\infty}^{+\infty}\left[\gamma \delta\left(t-t^{\prime}\right)+R\left(t-t^{\prime}\right)\right] G\left(t^{\prime}\right) d t^{\prime}
$$

where $\gamma \delta(t)$ is the instantaneous electronic response and and $R(t)$ is the nuclear response, see equation (1). $G(t)$ is the instrumental function accounting for the finite time resolution determined by the time width of the pump and probe pulses. Under specific conditions ${ }^{64}, G(t)$ corresponds to the temporal cross-correlation of the laser pulse intensities.

Instrumental function measurements. The measurement of the real instrumental function $G(t)$ is mandatory to extract the true OKE response of water, but it is far from trivial. By definition, the instrumental function in a HD-OKE experiment is the signal measured in a system with response function $R(t) \propto \delta(t)$, as directly inferred from equation (4). In practice, this condition can be fulfilled by choosing a sample with negligible or instantaneous nuclear response. In most of the papers published on this subject in recent years, a fused silica plate has been used for this purpose, as its nuclear contribution is considered negligible. In reality, silica presents a fast and weak, but not null, nuclear contribution that prevents from measuring the real instrumental function. Alternatively, the instrumental function has been obtained by measuring the second harmonic cross-correlation of the laser pulses ${ }^{48}$. This procedure, however, implicates some changes and adjustments in the OKE set-up that potentially modify the response function. In fact, any even small changes of the experimental conditions (for example, a different beam alignment or a modified angle of superposition of the beams inside the sample) affect the instrumental function. Therefore, any method for obtaining the instrumental function, which involves changes of the set-up, is unsafe and can yield an incorrect time profile. In other HD-OKE investigations, the response function has been obtained simply by fitting the electronic contribution to the HD-OKE signal with an analytic peak function (such as Gaussian, hyperbolic secant and so on). None of these methods enable the measurement of response function with the accuracy required by $\mathrm{HD}-\mathrm{OKE}$ investigations in the water sample, which is characterized by a fast- and low-level signal.

We extracted the instrumental function by performing HD-OKE measurements in a reference sample. This procedure allows us to accurately preserve the experimental conditions used during the water measurements. As the reference sample, we chose a plate of calcium fluoride $\left(\mathrm{CaF}_{2}\right)$. This a cubic ionic crystal, which is optically isotropic and, in the frequency range probed in our experiment, has only one Raman active band at $322 \mathrm{~cm}^{-1}$, due to the optical phonon $T_{2 \mathrm{~g}}$ symmetry. The calcium fluoride plate was dipped inside a water vial, identical to that used for water measurements, supported by the same sample holder.

The measurements of the instrumental function were done by just replacing the water vial with the water- $\mathrm{CaF}_{2}$ vial, leaving the rest of the set-up unchanged. 
The thickness of the $\mathrm{CaF}_{2}$ plate, $3 \mathrm{~mm}$, was enough to fully contain the probe and pump overlap volume, avoiding any spurious signal contribution by the outer water. We took a reference measurement for each set of water data. Maximum attention was taken to preserve the experimental conditions between the water and the reference measurement.

Supplementary Fig. S2 reports a typical HD-OKE signal obtained in the $\mathrm{CaF}_{2}$ reference sample. We performed a least-square fitting of the HD-OKE data of the reference sample with the simulated signal, according to equation (4), where the nuclear response is a single harmonic damped oscillator. We found that the instrumental function requires the sum of several analytical functions, typically a combination of Gaussian, Lorentzian and/or hyperbolic secant functions. In Supplementary Fig. S2, we report the instrumental function obtained by the iterative fitting procedure (continuous line) and the one obtained by fitting the electronic peak with a simple hyperbolic secant function (dashed line). Apparently, these two instrumental functions are very similar; however, the small differences cannot be neglected for an accurate investigation of water-fast HD-OKE response. This is mostly evident when the data are Fourier transformed to the frequency domain. In fact, obtaining the imaginary part of the frequency-dependent response from the time domain data involves the division by the Fourier transform of the instrumental function. Supplementary Fig. S3 shows the comparison between the response calculated using the instrumental function obtained by the iterative fitting procedure (continuous line) and the one obtained by simply fitting the electronic peak with a hyperbolic secant function (dashed line). We clearly see that only an accurate measurement of the real time domain instrumental function yields the correct response.

To our knowledge, this aspect of the OKE data analysis has never been addressed in such a quantitative way, and the present level of accuracy in analysing the role of the instrumental function in HD-OKE experiments is unprecedented in recent literature. In particular, we want to stress that the knowledge of the correct instrumental function is fundamental to extract the true OKE response, especially for the short delay in time range. This is particularly critical for weak signals characterized by complex relaxation dynamics, as in the case of water.

Data analysis and fits. According to equation (3), in our analysis of the HD-OKE data we are decomposing the electronic polarizability correlator, $\Phi_{\chi \chi}$, into the sum of $\Phi_{\mathrm{i}}(t)$ correlators. It is implicit that the individual slave correlators cannot be directly associated to specific physical variables. Each of these correlators in fact describes an 'average collective mode' whose dynamics is described by the SMC equations. The vibrational and relaxation features and coupling processes are included in the SMC equations by definition. In this respect, the present SMC equations represent a robust physical model capable of describing a complex dynamics, including vibrational and structural relaxations, implicitly accounting for their mutual coupling. In contrast to other approaches, the method does not require any decoupling or dynamic separation between the fast (vibrational) and the slow (relaxation) processes. Thus, once the temporal relaxation function of the master correlator is known, it can be used to calculate the relaxation of the slave correlators and, eventually, that of the OKE signal.

We found that the OKE data can be described in the entire temperature range by employing three slave correlators at most. In particular, the weight of the highest frequency contribution decreases monotonically with increasing temperature, becoming negligible at the two highest temperatures, where only two slave correlators are sufficient to reproduce the data. The parameters of the model are 16: the master equation parameters $\eta_{\mathrm{m}}, \Omega_{\mathrm{m}}, v_{1}$ and $v_{2}$, the slave equations parameters $\eta_{\mathrm{i}}, \Omega_{\mathrm{i}}, v_{i}^{\mathrm{s}}$ with $i=1,2,3$ and, finally, the three correlator amplitudes $a_{1}, a_{2}$ and $a_{3}$. The integro-differential equations were solved numerically with a step-by-step second order Runge-Kutta algorithm. Preliminary series of fits provided a qualitative estimate of the temperature behaviour of the parameters. In agreement with similar analyses reported in the literature ${ }^{56,66-68}$, in the subsequent step we forced some of the master parameters either to assume fixed values or to follow simple temperature trends, leaving all the slave parameters free. In Supplementary Fig. S4, we show the temperature dependence of the slave frequencies $\Omega_{\mathrm{i}}$ and the friction parameters $\eta_{\mathrm{m}}$ and $\eta_{\mathrm{i}}$, and of the three vertices $v_{i}^{\mathrm{s}}$. The temperature behaviour of the fitting parameters is consistent with the results obtained by the previous studies in glass-forming liquids ${ }^{56,66-68}$.

\section{References}

1. Mishima, O. \& Stanley, H. The relationship between liquid, supercooled and glassy water. Nature 396, 329-335 (1998).

2. Debenedetti, P. \& Stanley, H. E. Supercooled and glassy water. Phys. Today 56, 40-46 (2003).

3. Debenedetti, P. Supercooled and glassy water. J. Phys. Cond. Matter 15, R1669-R1726 (2003).

4. Soper, A. Structural transformations in amorphous ice and supercooled water and their relevance to the phase diagram of water. Mol. Phys. 106, 2053-2076 (2008).

5. Angell, C. A. Insights into phases of liquid water from study of its unusual glass-forming properties. Science 319, 582-587 (2008).

6. Nilsoon, A. \& Pettersson, L. Perspective on the structure of liquid water. Chem. Phys. 389, 1-34 (2011).
7. Moore, E. B. \& Molinero, V. Growing correlation length in supercooled water. J. Chem. Phys. 130, 244505 (2009)

8. Moore, E. B. \& Molinero, V. Structural transformation in supercooled water controls the crystallization rate of ice. Nature 479, 506-508 (2011).

9. Limmer, D. T. \& Chandler, D. The putative liquid-liquid transition is a liquidsolid transition in atomistic models of water. J. Chem. Phys. 135, 134503 (2011).

10. Sciortino, F., Saika-Voivod, I. \& Poole, P. H. Study of the ST2 model of water close to the liquid-liquid critical point. Phys. Chem. Chem. Phys. 13, 19759-19764 (2011).

11. Liu, Y., Palmer, J. C., Panagiotopoulos, A. Z. \& Debenedetti, P. G. Liquid-liquid transition in ST2 water. J. Chem. Phys. 137, 214505 (2012).

12. Kesselring, T. A., Franzese, G., Buldyrev, S. V., Herrmann, H. J. \& Stanley, H. E. Nanoscale dynamics of phase flipping in water near its hypothesized liquidliquid critical point. Sci. Rep. 2, 474 (2012).

13. Gallo, P. \& Sciortino, F. Ising universality class for the liquid-liquid critical point of a one component fluid: A finite-size scaling test. Phys. Rev. Lett. 109, 177801 (2012).

14. Poole, P. H., Bowles, R. K., Saika-Voivod, I. \& Sciortino, F. Free energy surface of ST2 water near the liquid-liquid phase transition. J. Chem. Phys. 138, 034505 (2013).

15. Poole, P. H., Sciortino, F., Essmann, U. \& Stanley, H. E. Phase behaviour of metastable water. Nature 360, 324-328 (1992).

16. Taschin, A., Cucini, R., Bartolini, P. \& Torre, R. Temperature of maximum density of water in hydrophilic confinement measured by transient grating spectroscopy. Europhys. Lett. 92, 26005 (2010).

17. Cucini, R., Taschin, A., Bartolini, P. \& Torre, R. Acoustic, thermal and flow processes in a water filled nanoporous glass by time-resolved optical spectroscopy. J. Mech. Phys. Solids 58, 1302-1317 (2010).

18. Gallo, P., Rovere, M. \& Chen, S.-H. Dynamic crossover in supercooled confined water: understanding bulk properties through confinement. J. Phys. Chem. Lett. 1, 729 (2010).

19. Mancinelli, R., Bruni, F. \& Ricci, M. Controversial evidence on the point of minimum density in deeply supercooled confined water. J. Phys. Chem. Lett. 1, 1277 (2010).

20. Taschin, A., Bartolini, P., Marcelli, A., Righini, R. \& Torre, R. A comparative study on bulk and nanoconfined water by time-resolved optical Kerr effect spectroscopy. Faraday Discuss. doi:10.1039/C3FD00060E (2013).

21. Mallamace, F. et al. Evidence of the existence of the low-density liquid phase in supercooled, confined water. Proc. Natl Acad. Sci. 104, 424-428 (2007).

22. Krishnamurthy, S., Bansil, R. \& Wiafe-Akenten, J. Low-frequency Raman spectrum of supercooled water. J. Chem. Phys. 79, 5863-5870 (1983).

23. Aliotta, F. et al. Role of $\mathrm{H}$ bond and cooperative effects in normal and supercooled water studied by anisotropic low frequency light scattering. J. Chem. Phys. 84, 4731-4738 (1986).

24. Walrafen, G., Fisher, M., Hokmabadi, M. \& Yang, W.-H. Temperature dependence of the low- and high-frequency Raman scattering from liquid water. J. Chem. Phys. 85, 6970-6982 (1986).

25. Rousset, J. L., Duval, E. \& Boukenter, A. Dynamical structure of water: low-frequency Raman scattering from a disordered network and aggregates. J. Chem. Phys. 92, 2150-2154 (1990).

26. Mizoguchi, K., Hori, Y. \& Tominaga, Y. Study on dynamical structure in wate and heavy water by low-frequency Raman spectroscopy. J. Chem. Phys. 97, 1961-1968 (1992).

27. Sokolov, A., Hurst, J. \& Quitmann, D. Dynamics of supercooled water: modecoupling theory approach. Phys. Rev. B 51, 12865-12868 (1995).

28. Torre, R. Time-Resolved Spectroscopy in Complex Liquids, an Experimental Perspective (Springer, 2008).

29. Torre, R., Bartolini, P. \& Righini, R. Structural relaxation in supercooled water by time-resolved spectroscopy. Nature 428, 296-298 (2004).

30. Taschin, A., Bartolini, P., Eramo, R. \& Torre, R. Supercooled water relaxation dynamics probed with heterodyne transient grating experiments. Phys. Rev. E 74, 031502 (2006).

31. Bartolini, P., Taschin, A., Eramo, R., Righini, R. \& Torre, R. Optical Kerr effect measurements on supercooled water: the experimental perspective. J. Phys. Conf. Series 177, 012009 (2009).

32. Taschin, A., Cucini, R., Bartolini, P. \& Torre, R. Does there exist an anomalous sound dispersion in supercooled water? Philos. Mag. 91, 1796-1800 (2011).

33. Castner, E., Chang, Y., Chu, Y. \& Walrafen, G. The intermolecular dynamics of liquid water. J. Chem. Phys. 102, 653-659 (1995).

34. Palese, S., Mukamel, S., Miller, R. \& Lotshaw, W. Interrogation of vibrational structure and line broadening of liquid water by Raman-induced Kerr effect measurements within multimode Brownian oscillator model. J. Phys. Chem. 100, 10380-10388 (1996).

35. Winkler, K., Lindner, J. \& Vohringer, P. Low-frequency depolarized Ramanspectral density of liquid water from femtosecond optical Kerr-effect 
measurements: lineshape analysis of restricted translational modes. Phys. Chem. Chem. Phys. 4, 2144-2155 (2002).

36. Ratajska-Gadomska, B., Bialkowski, B., Gadomski, W. \& Radzewicz, C. Ultrashort memory of the quasicrystalline order in water by optical Kerr effect spectroscopy. Chem. Phys. Lett. 429, 575-580 (2006).

37. Sonoda, M., Vechi, S. \& Skaf, M. A simulation study of the optical Kerr effect in liquid water. Phys. Chem. Chem. Phys. 7, 1176-1180 (2005).

38. Righini, R. Ultrafast optical Kerr effect in liquids and solids. Science 262, 1386-1390 (1993)

39. Hunt, N. T., Jaye, A. A. \& Meech, S. R. Ultrafast dynamics in complex fluids observed through the ultrafast optically-heterodyne-detected optical-Kerr-effect (OHD-OKE). Phys. Chem. Chem. Phys. 9, 2167-2180 (2007).

40. Bartolini, P., Taschin, A., Eramo, R. \& Torre, R. Optical Kerr Effect Experiments on Complex Liquids, A Direct Access to Fast Dynamic Processes. chap. 2, 73-127 (Springer, 2008).

41. Bartolini, P., Ricci, M., Torre, R., Righini, R. \& Santa, I. Diffusive and oscillatory dynamics of liquid iodobenzene measured by femtosecond optical Kerr effect. J. Chem. Phys. 110, 8653-8662 (1999).

42. Torre, R., Bartolini, P. \& Pick, R. Time-resolved optical Kerr effect in a fragile glass-forming liquid, Salol. Phys. Rev. E 57, 1912-1920 (1998).

43. Torre, R., Bartolini, P., Ricci, M. \& Pick, R. Time-resolved optical Kerr effect on a fragile glass-forming liquid: test of different mode-coupling theory aspects. Europhys. Lett. 52, 324-329 (2000).

44. Prevosto, D. et al. Relaxation processes in a epoxy resin studied by time resolved optical Kerr effect. Phys. Rev. E 66, 11502 (2002).

45. Ricci, M., Bartolini, P. \& Torre, R. Fast dynamics of a fragile glass-former by time resolved spectroscopy. Philos. Mag. B 82, 541-551 (2002).

46. Ricci, M., Wiebel, S., Bartolini, P., Taschin, A. \& Torre, R. Time-resolved optical Kerr effect experiments on supercooled benzene and test of modecoupling theory. Philos. Mag. 84, 1491-1499 (2004).

47. Fecko, C., Eaves, J. \& Tokmakoff, A. Isotropic and anisotropic Raman scattering from molecular liquids measured by spatially masked optical Kerr effect spectroscopy. J. Chem. Phys. 117, 1139-1154 (2002).

48. Kinoshita, S., Kai, Y., Yamaguchi, M. \& Yagi, T. Direct comparison between ultrafast optical Kerr effect and high-resolution light scattering spectroscopy Phys. Rev. Lett. 75, 148-151 (1995).

49. Skaf, M. \& Sonoda, M. Optical Kerr effect in supercooled water. Phys. Rev. Lett. 94, 137802 (2005)

50. Padró, J. A. \& Martí, J. Response to 'Comment on an interpretation of the lowfrequency spectrum of liquid water'. J. Chem. Phys. 120, 1659-1660 (2004).

51. DeSantis, A., Ercoli, A. \& Rocca, D. Comment on 'An interpretation of the lowfrequency spectrum of liquid water'. J. Chem. Phys. 120, 1657-1658 (2004).

52. Cho, M., Fleming, G. R., Saito, S., Ohmine, I. \& Stratt, R. M. Instantaneous normal mode analysis of liquid water. J. Chem. Phys. 100, 6672-6683 (1994).

53. Huang, C. et al. Increasing correlation length in bulk supercooled $\mathrm{H}_{2} \mathrm{O}, \mathrm{D}_{2} \mathrm{O}$, and $\mathrm{NaCl}$ solution determined from small angle $\mathrm{X}$-ray scattering. J. Chem. Phys. 133, 134504 (2010).

54. Götze, W. \& Sjögren, L. Relaxation processes in supercooled liquids. Rep. Progr. Phys. 55, 241-376 (1992).

55. Götze, W. \& Voigtmann, T. Universal and non-universal features of glassy relaxation in propylene carbonate. Phys. Rev. E 61, 4133-4147 (2000)

56. Götze, W. \& Sperl, M. Nearly-logarithmic decay of correlations in glassforming liquids. Phys. Rev. Lett. 92, 105701 (2004).

57. Götze, W. Complex Dynamics of Glass-Forming Liquids, a Mode-Coupling Theory (Oxford University Press, 2009).

58. Suzuki, Y., Takasaki, Y., Tominaga, Y. \& Mishima, O. Low-frequency Raman spectra of amorphous ices. Chem. Phys. Lett. 319, 81-84 (2000).
59. Nilsson, A., Huang, C. \& Pettersson, L. G. Fluctuations in ambient water. J. Mol. Liq. 176, 2-16 (2012).

60. Overduin, S. D. \& Patey, G. N. Understanding the structure factor and isothermal compressibility of ambient water in terms of local structural environments. J. Phys. Chem. B 116, 12014-12020 (2012).

61. Giraud, G., Gordon, C., Dunkin, I. \& Wynne, K. The effects of anion and cation substitution on the ultrafast solvent dynamics of ionic liquids: a time-resolved optical Kerr-effect spectroscopic study. J. Chem. Phys. 119, 464-477 (2003).

62. Bartolini, P., Eramo, R., Taschin, A., Pas, M. D. \& Torre, R. A real-time acquisition system for pump-probe spectroscopy. Philos. Mag. 87, 731-740 (2007).

63. Hellwarth, R. W. Third-order optical susceptibilities of liquids and solids. Prog Quant. Electr. 5, 1-68 (1977).

64. McMorrow, D., Lotshaw, W. \& Kenney-Wallace, G. Femtosecond optical Kerr studies on the origin of the non-linear responses in simple liquids. IEEE J. Quant. Electron. QE-24, 443-454 (1988).

65. Torre, R., Santa, I. \& Righini, R. Pre-transitional effects in the liquid-plastic phase transition of p-Terphenyl. Chem. Phys. Lett. 212, 90-95 (1993).

66. Alba-Simionesco, C. \& Krauzman, M. Low frequency Raman spectroscopy of supercooled fragile liquids analyzed with schematic mode coupling models J. Chem. Phys. 102, 6574-6585 (1995).

67. Krakoviack, V. \& Alba-Simionesco, C. What can be learned from the schematic mode-coupling approach to experimental data? J. Chem. Phys. 117, 2161-2171 (2002).

68. Wiebel, S. \& Wuttke, J. Structural relaxation and mode coupling in a nonglassforming liquid: depolarized light scattering in benzene. N. J. Phys. 4, 1-17 (2002).

\section{Acknowledgements}

The research has been performed at LENS. This work was supported by the REGIONE TOSCANA POR-CRO-FSE 2007-2013 by the EC COST Action MP0902-COINAPO and by the PRIN 2010/2011N.2010ERFKXL. We thank A. Marcelli for helpful discussion. We acknowledge M. De Pas, A. Montori and M. Giuntini for providing their continuous assistance in the set-up of the electronics. We also thank R. Ballerini and A. Hajeb for the accurate mechanical realizations.

\section{Author contributions}

The contribution from all the authors was fundamental to realize this study. More specifically: A.T., P.B., R.E. and R.T. planned the experiments; A.T., P.B., R.E. performed the experiments and analysed data; R.R. and R.T. interpreted the data and write the paper in collaboration with the other authors.

\section{Additional information}

Supplementary Information accompanies this paper at http://www.nature.com/ naturecommunications

Competing financial interests: The authors declare no competing financial interests.

Reprints and permission information is available online at http://npg.nature.com/ reprintsandpermissions/

How to cite this article: Taschin, A. et al. Evidences of two distinct local structures of water from ambient to supercooled conditions by optical Kerr effect experiments. Nat. Commun. 4:2401 doi: 10.1038/ncomms3401 (2013). 